\title{
Characteristics of Cirrhosis Patients With Left Ventricular Diastolic Dysfunction In China: A Single- Center Retrospective Study.
}

Jingjing Hu

Third People's Hospital of Hangzhou

Zhaobin Cai (D Hujj951009@163.com )

Third People's Hospital of Hangzhou

\section{Research Article}

Keywords: Left ventricular diastolic dysfunction, Liver cirrhosis, Cirrhotic cardiomyopathy

Posted Date: August 31st, 2021

DOl: https://doi.org/10.21203/rs.3.rs-818578/v1

License: (c) (i) This work is licensed under a Creative Commons Attribution 4.0 International License.

Read Full License 


\section{Abstract}

Objective: Mounting evidence links cirrhosis patients with left ventricular diastolic dysfunction邓LVDDQ has a poor prognosis. However, little is known about these particular individuals. Therefore, we conducted this cross-sectional study to assess the prevalence of LVDD and its associated risk factors.

Methods: Consecutive cirrhosis patient who were attending Hangzhou Xixi hospital from January 2018 to December 2019 were included in this study. According to the American Society of Echocardiography 2016 criteria, cirrhosis patients were sorted into two group: the left ventricular diastolic dysfunction(LVDD) group and left ventricular diastolic function normal(LVDDn) group. Patients' demographic data, clinical characteristics, laboratory data were recorded. Furthermore, we conducted a multi-factor analysis.

Results: A total of 398cirrhosis patients were included in the study. The incidence of LVDD in this study was $49.7 \%$ (198 cases). In this study, the mean age and BMI of the patients were $52.9 \pm 8.2$ years and $23.0 \pm 3.3 \mathrm{~kg} / \mathrm{m}^{2} \rrbracket$ respectively. Of 398 patients, $255(64.1 \%)$ of them were males. With regard to etiology, there were 296 patients (74.4\%) with hepatitis B cirrhosis and 59 patients $(14.8 \%)$ with alcoholic cirrhosis. The LVDD group had higher age, higher BMI, greater frequency of ascites and esophageal varices, prolonged prothrombin time, increased international normalized ratio, increased bilirubin, increased CK and AST, and longer QT interval than the LVDDn group $(p<0.05$, both). In terms of echocardiography, the LVDD group had larger aortic inner diameter, left atrial inner diameter and left ventricular wall diastolic thickness than LVDDn group $(p<0.05$, both). The multivariate analysis showed age $>55$ years, $\mathrm{BMI}>24 \mathrm{~kg} / \mathrm{m} 2$, hepatic decompensated, QTcB $>440 \mathrm{~ms}$ were independently associated with risk of LVDD.

Conclusion: The prevalence of LVDD among cirrhosis patients was 49.8\%. Cirrhosis patients with LVDD had worse liver function. Further, age $>55$ years, $B M 1>24 \mathrm{~kg} / \mathrm{m} 2$, hepatic decompensated, QTcB $>440 \mathrm{~ms}$ were independent predictors of LVDD.

\section{Background}

Hepatic fibrosis and liver cirrhosis are chronic disease and serious health problems worldwide. It is also known cirrhosis can damage other organs(such as the lung, kidney and heart), which has a tremendous impact on the quality of life and prognosis of patients ${ }^{1}$. It is reported that cirrhosis could lead to a hyperdynamic circulatory state, which manifests as an increase in mean arterial pressure, heart rate and a decrease in peripheral vascular resistance. It leads to structural and functional abnormalities of the heart ultimately. For many years, cardiac dysfunctions associated with liver cirrhosis has been attributed to the direct toxic effects of alcohol on the heart. Until recent years, many studies showed that circulatory dysfunction was secondary to liver cirrhosis and named it cirrhotic cardiomyopathy(CCM) ${ }^{23}$. The term CCM was defined as a spectrum of chronic cardiac dysfunction in cirrhotic patients in the absence of known heart disease, regardless of the etiology of cirrhosis. The pathogenesis of CCM includes diminished beta-adrenergic receptor signal transduction, cardiomyocyte cellular plasma membrane dysfunction, and increased activity or levels of cardio-depressant substances such as cytokines, 
endogenous cannabinoids, and nitric oxide ${ }^{4}$. Even though researchers were aware of CCM years ago. However, because patients with CCM have symptoms only during exercise or pharmacological stress ${ }^{5}$. The most important, the main causes of death in cirrhosis were gastrointestinal bleeding and hepatic encephalopathy. Hence, few clinicians will pay attention to CCM.

Recent evidence have shown that left ventricular diastolic dysfunction (LVDD) was identified as the most common and the first sign of cardiac abnormality among cirrhosis patients ${ }^{67}$. Furthermore, many researchers found LVDD is associated with poor prognosis of patients with cirrhosis. LVDD is associated with the risk of hepatorenal syndrome (HRS) ${ }^{8}$, septic shock ${ }^{9}$, and heart failure in the perioperative period following liver transplantation ${ }^{10}$. Therefore, LVDD is a very valuable diagnostic and prognostic tool for cirrhosis patients. Hence, we conducted this cross-sectional study to assess the prevalence of LVDD and its associated risk factors.

\section{Methods}

This was a cross-sectional study. We included all the cirrhosis patients who came to Hangzhou Xixi hospital between January 2018 and December 2019. Cirrhosis was diagnosed based on clinical and pathological findings, laboratory results, and diagnostic imaging. The exclusion criteria were: chronic renal disease, pregnancy, peripartum cardiomyopathy, hypertension, coronary artery disease, valvular heart disease, sick sinus syndrome/ pacemaker, thyroid dysfunction, portal vein thrombosis, transjugular intrahepatic portosystemic shunt insertion, hepatocellular carcinoma, and severe anemia.

All patients informed consent and signed the consent form. A full medical history was obtained from all subjects. Basic information including age, sex, blood pressure, BMI, medication history, etiology of liver disease, and history of hepatic encephalopathy, varices were record. Hemoglobin, creatinine, CK, CK-MB, Troponin I, prothrombin time, INR, and liver function tests including serum bilirubin, albumin, and transaminases were detected. Liver function was assessed based on the Child-Pugh scoring system. The liver-compensated stage is known as Child-Pugh $A$ and the liver-decompensated stage includes ChildPugh B and Child-Pugh C. Furthermore, transthoracic echocardiography and A12-lead surface EGG was obtained from all subjects. The transthoracic echocardiography was performed by two experienced operators. All patients were examined at rest in the left lateral decubitus position using a Vivid-9 ultrasound system (GE Healthcare, Chicago, IL, USA). LAD, right ventricular diameter(RVD), ventricular septal diastolic thickness(IVSDT), LWPWD, left ventricular end diastolic diameter(LVDd), left ventricular systolic diameter(LVSd), left ventricular ejection fraction(LVEF), left atrial volume index(LAVI), tricuspid regurgitation peak velocity $\left(T R_{\text {max }}\right), e^{\prime}\left(\right.$ early diastolic mitral annulus velocity), and $E / e^{\prime}$ (the ratio of $E$ and early filling wave) were assessed by the 2016 American Society of Echocardiography and European Association of Cardiovascular Imaging guidelines ${ }^{11}$. All parameters were recorded in three cardiac cycles and the mean of the measurements was taken for analysis. The criteria for LVDD were: (1)septum $e^{\prime}<7$ $\mathrm{cm} / \mathrm{s}$ and/or lateral wall $\mathrm{e}^{\prime}<10 \mathrm{~cm} / \mathrm{s}$; (2) mean E/e' > $14 \mathrm{~cm} / \mathrm{s}$; (3) LAVI > $34 \mathrm{ml} / \mathrm{m} 2$; (4) TR $\mathrm{TR}_{\max }>2.8 \mathrm{~m} / \mathrm{s}$. If more than two of these four parameters exceeded the cut-off value, the LVDD. A 12-lead surface EGG 
was obtained from all subjects in the supine position. The ECG was recorded at a paper speed of 25 $\mathrm{mm} / \mathrm{s}$. All measurements were made by one observer who was not aware of the patients' characteristics. We corrected QT was calculated using Bazett's formula and prolonged QT interval was considered to be present if QTC of more than 0.44 . The trial was approved by the Institutional Review Board of Hangzhou Xixi Hospital and was performed following the Declaration of Helsinki.

\section{Statistical analysis}

The sample size is calculated using the formula $\mathrm{N}=\frac{\mu_{\alpha / 2}^{2} \pi(1-\pi)}{\delta^{2}}$ and a is the significance level, $\pi$ is the overall rate and $\delta$ is the tolerance error. Based on the literature and the results of the pre-experiment, $\pi=$ $50 \%, a=0.05$ and $\delta=5 \%$ were set in this cross-sectional study. Therefore, at least 385 patients with liver cirrhosis need to be included in this study. Percentages and means \pm standard deviations are used to express the categorical and continuous variables, respectively. Student-t test was used to compare continuous variables. Categorical variables were analyzed using the Chi-square test. Logistic regression was used to determine the factors associated with LVDD. Age $>55$ years, BMl $>24 \mathrm{~kg} / \mathrm{m} 2$, history of alcoholics, history of oesophagogastric varices, liver-decompensated stage and prolonged QT interval were included in multivariate logistic regression. Significance was set at $P$ less than 0.05 . The statistical analysis was carried out using SPSS 20 and images was drawn by GraphPad Prism 9.0.

\section{Result}

\section{Patient characteristics}

A total of 398 cirrhosis patients were recruited. Patients were divided into the LVDD group and the the LVDDn group according to echocardiography. Table 1 shows the baseline characteristics of patients. Of the 398 patients, $198(49.7 \%)$ had LVDD. There was a significant difference in the mean age $(p<0.001)$ and mean BMI(0.044) among groups. Patients who had LVDD were older and heavier than those who without LVDD. Besides, patients with LVDD are more likely to have ascites $(p<0.01)$, esophageal and gastric varices $(p=0.05)$. Therefore, LVDD patients were more likely to use diuretic $(p<0.001)$ and beta blocks $(p=0.005)$. There was no significant difference in gender distribution, alcoholic cirrhosis, heart rate, SBP, DBP, history of smoke, history of alcoholics between two groups $(p>0.05$, both).

For laboratory investigations, HB, PT, INR, Creatinine, Bilirubin, AST, CK, Child-Pugh score, QTc were significantly different among the two groups $(p<0.05$, both). The HB and Bilirubin levels of the LVDD group were lower than the normal group. The levels of else were higher in the LVDD group. There was no significant difference in albumin, ALT, CK-MB, Troponin I between the two groups.

For echocardiography, Aortic dimension, LAD and LWPWD were significantly different among the two groups $(p<0.05$, both). The LVDD group had larger measurements. There was no significant difference in LVD, IVSDT, LVDd, LVSd, LVEF, FS between the two groups. 
Table 1

Base characteristics of patients with cirrhosis

\begin{tabular}{|c|c|c|c|c|}
\hline Characteristics & ALL(n = 398) & $\operatorname{LVDD}(\mathrm{n}=198)$ & $\operatorname{NLVDD}(n=200)$ & $\mathbf{P}$ \\
\hline Age(years) & $52.9 \pm 8.1$ & $56.1 \pm 6.8$ & $49.8 \pm 8.2$ & $<0.001$ \\
\hline Gender(male/female) & $255 / 143$ & $118 / 80$ & $137 / 63$ & 0.064 \\
\hline $\mathrm{BMI}(\mathrm{kg} / \mathrm{m} 2)$ & $23.0 \pm 3.3$ & $23.3 \pm 3.1$ & $22.6 \pm 3.5$ & 0.028 \\
\hline Alcoholic cirrhosis, n(\%) & $59(14.8 \%)$ & $29(14.6)$ & $30(15 \%)$ & 1.000 \\
\hline Heart rate (bpm) & $76.8 \pm 11.4$ & $75.8 \pm 11.0$ & $77.6 \pm 11.6$ & 0.113 \\
\hline $\mathrm{SBP}(\mathrm{mmHg})$ & $121.7 \pm 10.7$ & $122.5 \pm 10.9$ & $120.6 \pm 10.5$ & $0 ., 082$ \\
\hline DBP (mmHg) & $70.8 \pm 8.9$ & $70.9 \pm 9.4$ & $70.6 \pm 8.5$ & 0.082 \\
\hline History of smoke, n(\%) & $161(40.5 \%)$ & $80(40.4 \%)$ & $81(40.5 \%)$ & 0.984 \\
\hline History of alcoholic, n(\%) & $153(38.4 \%)$ & $86(43.6 \%)$ & $67(34 \%)$ & 0.042 \\
\hline Diuretic use, $\mathrm{n}(\%)$ & $166(41.7 \%)$ & $97(49 \%)$ & $69(34.5 \%)$ & $<0.001$ \\
\hline Beta blockers use, n(\%) & $115(28.9 \%)$ & $77(38.9 \%)$ & $38(19 \%)$ & $<0.001$ \\
\hline Antiviral drugs use, $\mathrm{n}(\%)$ & $296(74.4 \%)$ & 142(71.7\%) & $154(77 \%)$ & 0.191 \\
\hline Ascites, n(\%) & $112(28.1 \%)$ & $73(36.9 \%)$ & $40(20.0 \%)$ & $<0.001$ \\
\hline Varices, n(\%) & $166(41.7 \%)$ & $97(49.0 \%)$ & $69(34.5 \%)$ & 0.003 \\
\hline $\mathrm{HB}(\mathrm{g} / \mathrm{L})$ & $128.3 \pm 24.5$ & $123.3 \pm 25.6$ & $133.2 \pm 22.3$ & $<0.001$ \\
\hline PT(s) & $13.4 \pm 2.3$ & $14.0 \pm 2.5$ & $12.8 \pm 1.9$ & $<0.001$ \\
\hline INR & $1.14 \pm 0.21$ & $1.20 \pm 0.23$ & $1.09 \pm 0.18$ & $<0.001$ \\
\hline Albumin(g/L) & $39.1 \pm 7.2$ & $36.9 \pm 6.8$ & $41.3 \pm 6.9$ & 0.001 \\
\hline Creatinine $(\mu \mathrm{mol} / \mathrm{L})$ & $71.9 \pm 17.0$ & $72.0 \pm 18.1$ & $72.0 \pm 16.0$ & 0.951 \\
\hline Bilirubin $(\mu \mathrm{mol} / \mathrm{L})$ & $29.0 \pm 38.7$ & $35.6 \pm 51.2$ & $23.0 \pm 18.2$ & 0.001 \\
\hline $\operatorname{ALT}(\mathrm{U} / \mathrm{L})$ & $35.1 \pm 43.8$ & $37.9 \pm 58.1$ & $32.9 \pm 22.8$ & 0.255 \\
\hline AST(U/L) & $46.8 \pm 55.8$ & $53.8 \pm 76.3$ & $40.3 \pm 18.7$ & 0.017 \\
\hline CK(U/L) & $104.8 \pm 78.1$ & $116.5 \pm 98.9$ & $91.8 \pm 46.6$ & 0.002 \\
\hline CK-MB(U/L) & $28.4 \pm 28.4$ & $28.5 \pm 26.7$ & $27.5 \pm 29.2$ & 0.715 \\
\hline Troponin I(mg/L) & $0.01 \pm 0.002$ & $0.01 \pm 0.002$ & $0.01 \pm 0.02$ & 0.471 \\
\hline Child-Pugh score & $6.2 \pm 1.7$ & $6.6 \pm 1.8$ & $5.8 \pm 1.5$ & $<0.001$ \\
\hline Child A & $278(69.8 \%)$ & $116(58.6 \%)$ & $162(81 \%)$ & NA \\
\hline Child B & $91(22.9 \%)$ & $63(31.8 \%)$ & $28(14 \%)$ & NA \\
\hline
\end{tabular}




\begin{tabular}{|lllll|}
\hline Characteristics & ALL $(n=398)$ & $\operatorname{LVDD}(n=198)$ & $\operatorname{NLVDD}(\mathbf{n}=200)$ & P \\
\hline Child C & $29(7.3 \%)$ & $19(9.6 \%)$ & $10(5 \%)$ & NA \\
QTc $(m s)$ & $437 \pm 26.6$ & $446.1 \pm 26.4$ & $429.1 \pm 24.2$ & $<0.001$ \\
Aortic dimension & $2.74 \pm 0.32$ & $2.86 \pm 0.31$ & $2.62 \pm 0.29$ & $<0.001$ \\
LAD & $3.18 \pm 0.45$ & $3.29 \pm 0.51$ & $3.07 \pm 0.36$ & $<0.001$ \\
RVD & $2.03 \pm 0.28$ & $2.05 \pm 0.30$ & $2.02 \pm 0.26$ & 0.169 \\
IVSDT & $0.88 \pm 0.13$ & $0.89 \pm 0.13$ & $0.86 \pm 0.13$ & 0.103 \\
LWPWD & $0.89 \pm 0.35$ & $0.94 \pm 0.47$ & $0.84 \pm 0.16$ & 0.005 \\
LVDd & $4.79 \pm 0.53$ & $4.82 \pm 0.57$ & $4.77 \pm 0.48$ & 0.352 \\
LVSd & $3.10 \pm 0.35$ & $3.08 \pm 0.31$ & $3.06 \pm 0.39$ & 0.673 \\
LVEF(\%) & $65.91 \pm 4.72$ & $66.32 \pm 4.36$ & $65.42 \pm 5.02$ & 0.055 \\
\hline
\end{tabular}

Factors associated with LVDD

On multivariate logistic regression of factors associated with LVDD, age (OR 1.128; 95\% $\mathrm{Cl} 1.089-1.169$; $p<0.001)$, BMI(OR 1.166; 95\% Cl 1.076-1.264; $p<0.001)$, Child score(OR 1.202; 95\% Cl 1.007-1.435; $p=$ $0.041)$ and prolong QTc (OR 2.171; $95 \% \mathrm{Cl} 1.232-3.828 ; p=0.007)$ were factors significantly associated with LVDD(see Table 2).

Table 2

Factors associated with LVDD

\begin{tabular}{|llll|}
\hline Factors & OR & $95 \% \mathrm{Cl}$ & $\boldsymbol{P}$ \\
\hline Age $>55$ years & 3.895 & $2.424-6.258$ & $<0.001$ \\
BMI $>24 \mathrm{~kg} / \mathrm{m}^{2}$ & 2.100 & $1.276-3.456$ & 0.003 \\
History of alcoholic & 1.699 & $0.905-3.192$ & 0.099 \\
Esophagogastric varices & 1.090 & $0.681-1.744$ & 0.719 \\
Liver-decompensated & 1.810 & $1.017-3.224$ & 0.044 \\
QTc $>440 \mathrm{~ms}$ & 2.382 & $1.436-3.951$ & 0.001 \\
\hline
\end{tabular}

\section{Discussion}

Cirrhosis induces a hyperdynamic circulation characterized by high cardiac output and increased cardiac work which may be latent clinically because of decreased afterload by reduced systemic vascular resistance. Heart failure may become clinically overt under strain or vasoconstrictors ${ }^{12}$. LVDD was an early marker of CCM. In a rest state, LVDD was the main characteristic of patients with cirrhosis. LVDD can be seen as an early manifestation of CCM. As an early manifestation, LVDD may occur before and 
partly contribute to the development of systolic dysfunction ${ }^{11}$. This study was designed to clarify the clinical characteristics and risk factors of cirrhosis patients with LVDD.

In our study, we found that the prevalence of LVDD in patients with cirrhosis is $49.7 \%$. According to different studies, the prevalence of LVDD in cirrhotic patients is different(20-80\%), ${ }^{6,15}$. This is likely the result of several factors, such as different sample sizes, different definitions of LVDD, different races, and not except confounding factors of cardiac dysfunction in study. Most of the previous studies have diagnosed LVDD based on the E/A ratio $<1$, DT $<200 \mathrm{~ms}$, or isovolumic relaxation time (IVRT) $>80 \mathrm{~ms}$. In our population, cirrhosis patients with LVDD had higher age( $56.1 \pm 6.8 \mathrm{vs.49.8} \pm 8.2 ; p<0.01)$ and higher $\operatorname{BMI}(23.3 \pm 3.3 v s .22 .7 \pm 3.5 ; p=0.044)$. Alexopoulou et al made similar observations ${ }^{16}$. Moreover, we observed patients with LVDD had a more severe liver function, some liver function indexes, such as varices, $\mathrm{PT}$, and bilirubin were worse in the LVDD group. It resulted in Child-Pugh score was higher in LVDD group( $6.5 \pm 1.8 v s .5 .7 \pm 1.4 ; p<0.001)$. This finding was also reported by Papastergiou et al. ${ }^{17}$,Merli et al. ${ }^{18}$, and Salari etal. ${ }^{19}$. Because ascites(36.9\%vs. $\left.19.5 \% ; p<0.01\right)$ and varies $(49 \%$ vs. $34.5 \% ; p=0.005)$ were more common in LVDD patients. Therefore, LVDD patients were more commonly use diuretic and beta-blockers. QTc prolongation was a typical characteristic of CCM. In our study, QTc prolongation was noted in 178(44.7\%) patients with LVDD. Also, QTc was found longer in cirrhosis patients with $\operatorname{LVDD}(446.1 \pm 26.4 \mathrm{vs} .429 .1 \pm 24.1 ; p<0.001)$. Barbosa et al. ${ }^{20}$ and Zambruni et al. ${ }^{21}$ observed the same result. The cardiac structural changes differences detected in patients with compared with those without LVDD included larger aortic dimension, larger $\operatorname{LAD}$, and larger $\operatorname{LWPWD}(p<0.05$, both $)$.

In multivariate logistic regression, the principal findings of this study increased age(OR $1.128,95 \% \mathrm{Cl}$ 1.089-1.169), BMI(OR 1.166, 95\% Cl 1.076-1.264), Child-Pugh score(OR 1.202, 95\% Cl 1.007-1.435) and QTc > 440(OR 2.171, 95\% Cl 1.232-3.828) were closely correlated with the risk of LVDD in cirrhosis individuals. Similarly, Cesari et al. ${ }^{6}$ discovered that age was associated with LVDD in the cirrhosis population. Besides, the LVM index was identified as the strongest predictor of LVDD in their study. It was well known that for subjects without a history of cardiovascular disease the risk of having diastolic dysfunction increases in older ${ }^{22}$. The relationship between the Child-Pugh score and LVDD has been controversial. The current study found that it was an independent risk factor for LVDD. This finding is contrary to previous studies which have suggested that the severity of cirrhosis is not related to LVDD $^{16,23-25}$. Similar to previous studies, we did not found patients with alcoholic cirrhosis were more likely to develop LVDD ${ }^{16,24,25}$. Surprisingly, QTC > 440ms was found to be a factor that relates to LVDD. Prolongation of the QT interval predisposes the patients to a potentially fatal polymorphic ventricular tachycardia called torsade de pointes, which can degenerate into ventricular fibrillation and cause sudden cardiac death ${ }^{26}$. This is close to the results presented by Bernardi et al. ${ }^{27}$. However, Zuberi et al. ${ }^{28}$ and Bhatti et al. ${ }^{3}$ found the frequency of QTc prolongation was much lower than us. This discrepancy may be explained by the presence of other compounding factors such as electrolyte disturbances, concomitant cardiac problems, or use of QTc prolonging drugs, which were excluded in our study but might have been included in other studies. Therefore, cirrhosis patients who were older, heavier, higher Child-Pugh score, and QTC $>440 \mathrm{~ms}$ should test ultrasonic cardiogram. 
The strength of our study was LVDD estimation using the latest diagnostic method. Besides, we excluded the cirrhosis patients which had factors affected the cardiac function. Last but not least, we had the largest sample size than previous studies. Several limitations have to be interpreted in the present study: first, this is a cross-sectional study that limited the actual relationship evaluation. Afterward, we will follow up on this population. Additionally, most patients with cirrhosis in our population had a mild liver function. It may have an impact on the result.

\section{Conclusions}

In summary, we observed the high prevalence of LVDD among cirrhosis patients. Most important of all, increase age(OR 1.128, 95\% Cl 1.089-1.169), BMI(OR 1.166, 95\% Cl 1.076-1.264), Child-Pugh score(OR $1.202,95 \% \mathrm{Cl} 1.007-1.435)$, and QTc $>440 \mathrm{~ms}$ (OR 2.171, 95\% $\mathrm{Cl} 1.232-3.828$ ) would be predictive markers for development LVDD in cirrhosis patients. Hence, we hold the opinion cirrhosis patient with older age, obesity, poor liver function and prolongation QTc need to do echocardiogram tests more frequently.

\section{Declarations}

\section{Acknowledgements}

None.

\section{Funding}

None.

\section{Availability of data and materials}

Relevant raw data from this study can be readily available to any scientist wishing to use them for noncommercial purposes per request from the authors. Please feel free to contact $\mathrm{Dr}$ Zhabin Cai at Hujj951009@163.com if someone wants to request the data.

\section{Authors' contributions}

Zhaobin Cai: study design; Jingjing Hu: data acquisition, data analysis, drafting of manuscript; All authors read and approved the final manuscript.

\section{Declaration of interest}

The authors report no conflicts of interest. The authors alone are responsible for the content and writing of the paper.

\section{Conset for publication}


Not applicable

\section{References}

1 Al-Hamoudi, W. K. Cardiovascular changes in cirrhosis: pathogenesis and clinical implications. Saudi J Gastroentero/ 16, 145-153, doi:10.4103/1319-3767.65181 (2010).

2 Kazankov, K. et al. Resting myocardial dysfunction in cirrhosis quantified by tissue Doppler imaging. Liver Int 31, 534-540, doi:10.1111/j.1478-3231.2011.02468.x (2011).

3 Bashir Bhatti, A., Ali, F. \& Akbar Satti, S. Prolonged QTc Interval Is an Electrophysiological Hallmark of Cirrhotic Cardiomyopathy. Open Journal of Internal Medicine 04, 33-39, doi:10.4236/ojim.2014.41006 (2014).

4 Lee, S. S. Cardiac abnormalities in liver cirrhosis. West J Med 151, 530-535 (1989).

5 Moller, S. \& Henriksen, J. H. Cirrhotic cardiomyopathy. J Hepato/ 53, 179-190, doi:10.1016/j.jhep.2010.02.023 (2010).

6 Cesari, M., Fasolato, S., Rosi, S. \& Angeli, P. Cardiac dysfunction in patients with cirrhosis: is the systolic component its main feature? Eur J Gastroenterol Hepatol 27, 660-666, doi:10.1097/MEG.0000000000000340 (2015).

7 Armstrong, M. J. et al. Diastolic dysfunction on echocardiography does not predict survival after transjugular intrahepatic portosystemic stent-shunt in patients with cirrhosis. Aliment Pharmacol Ther 49, 797-806, doi:10.1111/apt.15164 (2019).

8 Premkumar, M. et al. Left Ventricular Diastolic Dysfunction is Associated with Renal Dysfunction, Poor Survival and Low Health Related Quality of Life in Cirrhosis. Journal of clinical and experimental hepatology 9, 324-333, doi:10.1016/j.jceh.2018.08.008 (2019).

9 Prabhu, S. D. Cytokine-induced modulation of cardiac function. Circ Res 95, 1140-1153, doi:10.1161/01.RES.0000150734.79804.92 (2004).

10 Rahman, S. \& Mallett, S. V. Cirrhotic cardiomyopathy: Implications for the perioperative management of liver transplant patients. World J Hepatol 7, 507-520, doi:10.4254/wjh.v7.i3.507 (2015).

11 Nagueh, S. F. et al. Recommendations for the Evaluation of Left Ventricular Diastolic Function by Echocardiography: An Update from the American Society of Echocardiography and the European Association of Cardiovascular Imaging. European heart journal cardiovascular Imaging 17, 1321-1360, doi:10.1093/ehjci/jew082 (2016).

12 Carvalho, M. V. H., Kroll, P. C., Kroll, R. T. M. \& Carvalho, V. N. Cirrhotic cardiomyopathy: the liver affects the heart. Braz J Med Biol Res 52, e7809, doi:10.1590/1414-431X20187809 (2019). 
13 Hammami, R. et al. Cirrhotic cardiomyopathy: is there any correlation between the stage of cardiac impairment and the severity of liver disease? Libyan J Med 12, 1283162,

doi:10.1080/19932820.2017.1283162 (2017).

14 Farouk, H., Al-Maimoony, T., Nasr, A., El-Serafy, M. \& Ghany, M. A. Echocardiographic assessment of the left ventricular diastolic function in patients with non-alcoholic liver cirrhosis. Cor et Vasa 59, e540-e545, doi:10.1016/j.crvasa.2016.12.011 (2017).

15 Bokarvadia, R., Jain, M., Kedarisetty, C., Varghese, J. \& Venkataraman, J. Prevalence and clinical presentation of cirrhotic cardiomyopathy: A single centre experience from southern India. Indian J Gastroentero/ 38, 150-157, doi:10.1007/s12664-019-00946-7 (2019).

16 Alexopoulou, A. et al. Diastolic myocardial dysfunction does not affect survival in patients with cirrhosis. Transp/ Int 25, 1174-1181, doi:10.1111/j.1432-2277.2012.01547.x (2012).

17 Papastergiou, V. et al. Ultrasonographic prevalence and factors predicting left ventricular diastolic dysfunction in patients with liver cirrhosis: is there a correlation between the grade of diastolic dysfunction and the grade of liver disease? ScientificWorldJourna/ 2012, 615057, doi:10.1100/2012/615057 (2012).

18 Merli, M. et al. Cardiac dysfunction in cirrhosis is not associated with the severity of liver disease. Eur J Intern Med 24, 172-176, doi:10.1016/j.ejim.2012.08.007 (2013).

19 Salari, A., Shafaghi, A., Ofoghi, M., Saeidinia, A. \& Mansour-Ghanaei, F. Diastolic dysfunction and severity of cirrhosis in nonalcoholic cirrhotic patients. Int J Hepato/ 2013, 892876, doi:10.1155/2013/892876 (2013).

20 Barbosa, M. et al. Cirrhotic cardiomyopathy: Isn't stress evaluation always required for the diagnosis? World J Hepato/ 8, 200-206, doi:10.4254/wjh.v8.i3.200 (2016).

21 Zambruni, A. et al. QT interval correction in patients with cirrhosis. J Cardiovasc Electrophysio/ 18, 7782, doi:10.1111/j.1540-8167.2006.00622.x (2007).

22 Nagueh, S. F. et al. Recommendations for the Evaluation of Left Ventricular Diastolic Function by Echocardiography: An Update from the American Society of Echocardiography and the European Association of Cardiovascular Imaging. J Am Soc Echocardiogr 29, 277-314, doi:10.1016/j.echo.2016.01.011 (2016).

23 Karagiannakis, D. S., Vlachogiannakos, J., Anastasiadis, G., Vafiadis-Zouboulis, I. \& Ladas, S. D. Diastolic cardiac dysfunction is a predictor of dismal prognosis in patients with liver cirrhosis. Hepatol Int 8, 588-594, doi:10.1007/s12072-014-9544-6 (2014).

24 Somani, P. O., Contractor, Q., Chaurasia, A. S. \& Rathi, P. M. Diastolic dysfunction characterizes cirrhotic cardiomyopathy. Indian Heart J 66, 649-655, doi:10.1016/j.ihj.2014.06.001 (2014). 
25 Falletta, C. et al. Diastolic dysfunction diagnosed by tissue Doppler imaging in cirrhotic patients: Prevalence and its possible relationship with clinical outcome. Eur J Intern Med 26, 830-834, doi:10.1016/j.ejim.2015.10.009 (2015).

26 Schwartz, P. J. et al. Inherited cardiac arrhythmias. Nat Rev Dis Primers 6, 58, doi:10.1038/s41572-0200188-7 (2020).

27 M, B. et al. Q-T interval prolongation in cirrhosis: prevalence, relationship with severity, and etiology of the disease and possible pathogenetic factors. Hepatology 27, 28-34, doi:10.1002/hep.510270106 (1998).

28 Zuberi, B. F. et al. Comparison of heart rate and QTc duration in patients of cirrhosis of liver with noncirrhotic controls. J Coll Physicians Surg Pak 17, 69-71, doi:02.2007/JCPSP.6971 (2007). 